\title{
Behavior of the longitudinal structure function in NLO analysis at low $x$
}

\section{Gholam Reza BOROUN*广}

E-mail: grboroun@gmail.com

Behavior of the longitudinal structure function in the next- to- leading order of the perturbation theory at low $\mathrm{x}$, based on of the exponent $\lambda_{g}$ gluon distribution and $\lambda_{S}$ structure function from the Regge- like behavior at this limit is presented. This approach shows, the longitudinal structure function has the hard-Pomeron behavior. All the results can consistently be described within the framework of PQCD, which essentially shows increases as x decreases.

European Physical Society Europhysics Conference on High Energy Physics, EPS-HEP 2009, July 16 - 222009

Krakow, Poland

* Speaker.

${ }^{\dagger}$ A footnote may follow. 
In the next- to -leading order, the longitudinal structure function is proportional to $F_{2}=$ $x \sum_{j=1}^{N_{f}} e_{j}^{2}(q+\bar{q})_{j}, F_{2}^{S}=\left(\sum_{j=1}^{N_{f}} e_{j}^{2} / N_{f}\right) x \sum_{j=1}^{N_{f}}(q+\bar{q})_{j}$, and the gluon distribution $g$, respectively:

$$
\begin{aligned}
F_{L}\left(x, Q^{2}\right)= & \int_{x}^{1} \frac{d y}{y} K^{N S}\left(\frac{x}{y}, Q^{2}\right) F_{2}\left(y, Q^{2}\right)+\int_{x}^{1} \frac{d y}{y} K^{S}\left(\frac{x}{y}, Q^{2}\right) F_{2}^{S}\left(y, Q^{2}\right) \\
& +\int_{x}^{1} \frac{d y}{y} K^{G}\left(\frac{x}{y}, Q^{2}\right) G\left(y, Q^{2}\right),
\end{aligned}
$$

where $G\left(x, Q^{2}\right)=x g\left(x, Q^{2}\right)$ and $e_{j}$ are the quark charges and $N_{f}$ is the number of flavors[1-2]. In this work, we restrict our investigations to the Regge- like behavior for the gluon distribution and the structure function by the following forms:

$$
F_{2}\left(x, Q^{2}\right)=x S \sim A_{S} x^{-\lambda_{S}}, x g\left(x, Q^{2}\right)=A_{g} x^{-\lambda_{g}} .
$$

The singlet part of the structure function is controlled by Pomeron exchange at small $x$, where $\lambda_{S}$ is the Pomeron intercept minus one. The exponent was rapid rise in $Q^{2}$ of the structure function at this limit. This steep behavior of the structure function generates a similar steep behavior of the gluon distribution at small $x$, where $\lambda_{S} \neq \lambda_{g}$ in next- to- leading order analysis. We also note that $\lambda_{g}$ is the Pomeron intercept minus one and rises with $Q^{2}$ [3]. A set of formula to extract the gluon and the structure function exponents was given in Ref.4. On the basis of these results, we concentrate on the hard- Pomeron in our calculations and present an approximation analytical solution for the longitudinal structure function in the NLO corrections. We test its validity comparing it in NLO with that of H1 data [5], Donnachie \& landshoff [6], MVV[7] and attempt to see how the predictions for longitudinal structure function are compared with the experimental data, that our numerical predictions are presented as function of $x$ for the $Q^{2}=12,15,20$ and $25 \mathrm{GeV}^{2}$. So that, on the basis of Regge-like behavior, the longitudinal structure function is reduced to:

$$
F_{L}\left(x, Q^{2}\right)=\int_{x}^{1} \frac{d y}{y}\left[K^{N S}\left(\frac{x}{y}, Q^{2}\right)+K^{S}\left(\frac{x}{y}, Q^{2}\right)\right] A_{S} y^{-\lambda_{S}}+\int_{x}^{1} \frac{d y}{y} K^{G}\left(\frac{x}{y}, Q^{2}\right) A_{g} y^{-\lambda_{g}},
$$

where the nonsinglet quark density is negligible at small $x$ and the kernels $K^{i}(\mathrm{i}=$ nonsinglet, singlet and gluon) are given by Eqs.14-16 in Ref.8. These equations are a set of formulas to extract the longitudinal structure function, using the gluon distribution exponent and the structure function exponent determined at small $x$ in the next- to- leading order of the perturbation theory. Thus implying that Regge theory and perturbative evolution may be made compatible at small $x$. Thus this behavior at low $x$ is consistent with a dependence $F_{L}=A_{L} x^{-\lambda_{L}}$ that the power $\lambda_{L}$ is found to be $\sim 0.23$ and this value is corresponds to the hard pomeron intercept.

\section{References}

[1] J.L.Miramontes, J.sanchez Guillen and E.Zas, Phys.Rev.D 35, 863(1987).

[2] D.I.Kazakov, et.al., Phys.Rev.Lett. 65, 1535(1990).

[3] A.D.Martin, W.S.Striling and R.G.Roberts, Euro.J.Phys.C 23, 73(2002).

[4] G.R.Boroun and B.Rezaie, Phys.Atom.Nucl.vol.71, No.6, 1(2008).

[5] C.Adloff et.al, H1 Collab., Eur.Phys.J.C21, 33(2001). 

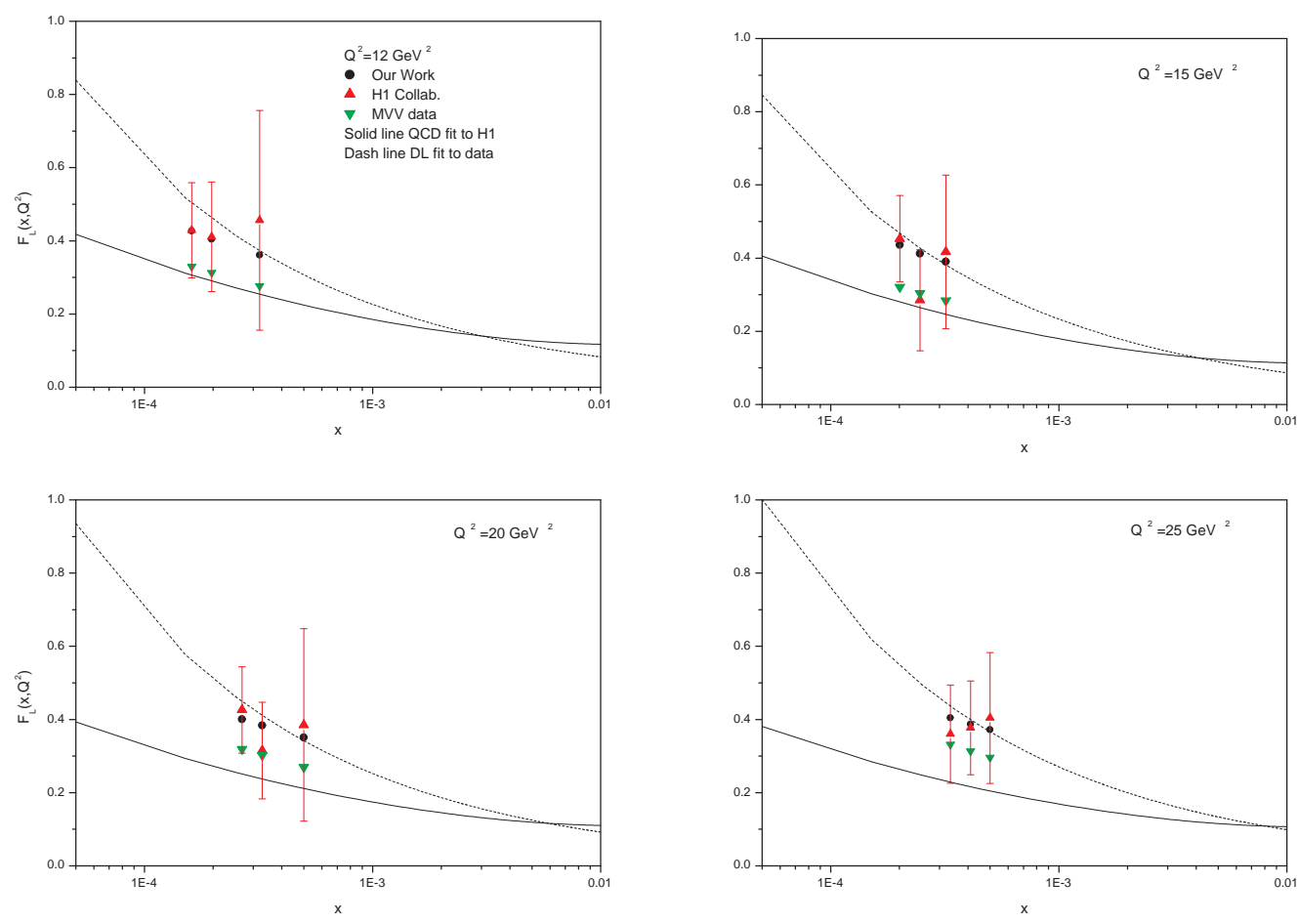

Figure 1: $\mathrm{H} 1$ data [5](up triangle) for the longitudinal structure function at $Q^{2}=12,15,20$ and $25 \mathrm{GeV}^{2}$ values, with our NLO data calculations. The error on the H1 data is the total uncertainty of the determination of $F_{L}$ representing the statistical, the systematic and the model errors added in quadrature. Down triangle data are the MVV prediction [7]. The solid line is the NLO QCD fit to the H1 data for $y<0.35$ and $Q^{2} \geq 3.5 \mathrm{GeV}^{2}$. The dash line is the DL [6] fit to $F_{L}$.

[6] A. Donnachie and P.V.Landshoff, Phys.Lett.B533, 277(2002); Phys.Lett.B550, 160(2002); J.R.Cudell, A. Donnachie and P.V.Landshoff, Phys.Lett.B448, 281(1999); P.V.Landshoff, hep-ph/0203084.

[7] S.Moch, J.A.M.Vermaseren and A.Vogt, Phys.Lett.B 606, 123(2005).

[8] G.R.Boroun, Int.J.Mod.Phys.E.18, No.1, 1(2009). 